\title{
Téoros
}

Revue de recherche en tourisme

\section{La technologie au service des entreprises touristiques : défis et opportunités}

\section{Robert Héroux}

Volume 14, numéro 3, automne 1995

Tourisme et technologies de l'information

URI : https://id.erudit.org/iderudit/1075078ar

DOI : https://doi.org/10.7202/1075078ar

Aller au sommaire du numéro

Éditeur(s)

Université du Québec à Montréal

ISSN

0712-8657 (imprimé)

1923-2705 (numérique)

Découvrir la revue

Citer cet article

Héroux, R. (1995). La technologie au service des entreprises touristiques : défis et opportunités. Téoros, 14(3), 6-7. https://doi.org/10.7202/1075078ar d'utilisation que vous pouvez consulter en ligne.

https://apropos.erudit.org/fr/usagers/politique-dutilisation/ 


\section{LA TECHNOLOGIE}

\section{AU SERVICE DES ENTREPRISES TOURISTIOUES:}

\section{DÉFIS ET OPPORTUNITÉS Robert IIéroux}

Robert Héroux

est directeur chez IBM

Canada Ltée.

(e-mail: heroux@ibm.net)

(http://www.ibm.com/internet)
L'industrle touristique vit actuellement une pérlode des plus florissantes. La mondlalisation des marchés, la prolifération d'accords commerclaux tels I'ALENA, alns| que l'émergence de nouveaux marchés ont eu pour effet de favorlser une crolssance du volume d'affalres pour le secteur tourlsUque. De plus. II est prévu que le cllmat économlque favorable se malntlendra au cours des prochalnes annés. A titre d'exemple, le revenu moyen quotidien par chambre dans le secteur hôteller augmentera plus rapidement que le laux dinflation (4,6\% versus $3,3 \%$ respectivement) aul cours des 5 prochaines années, et le taux d'occupation moyen devrait se maintenir à près de $70 \%$.

\section{Des clients plus exigeants et une compétition accrue}

Les voyageurs d'affalres et les consommateurs sont de plus en plus exigeants. Ce changement d'attitude peut s'expllquer de deux façons: Ia rationalisation des dépenses dans les entreprises a amené des politiques corporatives de voyage plus strictes où l'on essale de maximlser la valeur obtente pour chaque dollar dépensé et, deuxlèmement, par une compétition accrue entre les entreprises touristiques où I'on offre de plus en plus de services au voyageur d'affalres daus l'espolr de l'arracher à la compétition et de le ndêllser.

Cela a pour effet de créer le dllemme sulvant: comment offrir plus de services tout en malntenant ses couts au plus bas?

Une des réponses se trouve dans l'utilisation optimale des technologles de l'information. Employées effoncement, elles permettent d'optumlser les opérations d'une entreprise touristuque, d'améliorer la qualité de l'information pour la prise de décision et que de mleux comprendre et gérer les besolns de la clientèle afln d'obtenlr un avantage concurrentiel.

\section{La globalisation des marchés entraîne celle des technologies}

Les deux technologles de base assurant la distribution pour les entreprises touristiques furent et demeurent les centrales de réservations ("Global Distributlon Systems" ou GDS, et "Central Reservation Systems" ou CRS). Les deux secteurs connalssent une crolssance de leurs volumes de transactlons. Cependant, certaines tendances de ratlonalisat.lon des réseaux de distribution et l'émergence de canaux alternatifs tels Internet, America Online ou Prodlay. pourralent avolr pour effet de créer une certaine turbulence dans l'industrle et d'offrir de nouvelles possibilités à ceux qui sauront en tirer avantage.
Pour mleux comprendre la direction du marché, II est important de falre lol un bref rappel hlstorlque

\section{Réseaux de distribution (GDS)}

Les GDS ont connu trols phases de développement distinctes au cours des 30 dernlères années.

Au début des années 60 . Amerlcan Alrlines introdult Sabre sa centrale de réservation supportant ses vols et ses agents. Les autres compagnles emboittèrent, le pas.

Puls survint la deuxlème phase au mllleu des années 70 Iorsque Unlted Alrlines et American Alrlines réallsè rent que d'importantes économles pourralent être réallsées sl les agents de voyage ut.llsalent leurs systimes plutôt. que le téleéphone pour effectuer des réservations.

La trolslème phase se produlsit à la fin des années 80 Iorsque les compagnles aérlennes ont alouté la possiblité d'effectuer des réservatlons d'hôtels et de volture dans la même transactlon. La complexlté et l'Investlssement requis par cette amélloratlon susclta alors plusleurs fuslons parm! les GDS.

Au|ourd'luul, quatre réseaux majeurs luttent pour se positionner avantagensement pour les prochalnes années: Sabre, Worldspan. Galleo Intermational et Amadeus. Leur objectif demeure d'effectuer le nombre le plus élevé de réservatlons de slèges, de chambres et de voltures. Pour ce falre, leurs stratégles se résument en chnq points:

1) maximiser le nombre dagents de voyages ut.llisant leurs services.

2) exlger une compensatlon des hôtellers pour chaque réservation falte vla leurs services,

3) offrir un encouragement pour l'utilsation de leurs servlces,

4) obtenir un acces direct aux plus grandes entreprlses,

5) permettre aux consommateurs de falre leurs propres réservatlons en utllisant un PC et un modem.

\section{Centrale de réservation (CRS)}

Les premlères centrales de réservation dans le secteur hôteller virent le jour vers le milleu des années 60. Depuls, une multitude de développements survinrent et la majorité des activités se situent aujourd'hul dans les secteurs sulvants:

- I'amélioration et la consolidation des centrales par les plus grandes chaînes hotellères.

- Ia dlsponibilité de logiciels de réservation sur le marché permettant aux plus petites chaînes l'établissement de leurs propres centres de réservatlon. 
- les réseaux de distrlbution des compagnles aérlennes offrent un Interface et un acces plus efflcace aux cen= trales de réservation des hôtels.

- des entreprises Indépendantes offrent des servlces de réservation aux hôtels ne voulant pas engager leurs piopres ressources dans ce secteur d'activité.

Peu Importe l'approche cholsle, les centrales de réservation ont des caractérlstiques communes:

1) melleur contrôle sur la disponibilité des chambres

2) gestion du prix des chambres

3) accès simple et sécurltalre à des tarlfs pré-négoclés.

4) accès à une banque de chambres.

5) rapports de ventes plus sophlstiqués,

6) meilleure gestlon des groupes et forfalts.

7) Identuflcation des grands voyageurs et gestion plus effcace des programmes de fidéllsation.

Enfin, une dernlère composante technologlque permet de reller les centrales de réservation (CRS) aux réseaux de dlstrlbution (GDS): les services d'interconnectlvité (plus communément appelés "Universal Switch"). Des compagntes tels que Thisco ou Wizcom fouent ce rôle.

\section{Une profitabilité accrue via la technologie}

Il est. notoire que la profitabilité est élablie par la différence entre les revenus et les dépenses. On a longtemps perçu la technologle comme un outll pour rédulre ou exercer un melleur contrôle sur les côtts, et délalssé la partle revenu de l'équation. Límportance accrue accordée aux outils de gestion de la fidélisation ouvre cependant de nouveaux horlzons.

Pour Illustrer comment la technologle peut être utllisée afln de maximiser les revenus d'une entreprise tonristique, nous concentrerons notre propos sur le segment hotteller:

Trols Indicateurs économlques sont couramment ut.llisés pour mesurer le nlveau de revenus dans ce secteur: le revenu moyen quotidlen par chambre, le taux d'occupation, alns! qu'un Indice relativement, nouveau qul est le résultat de la multiplication des deux autres, solt le revenu par chambre disponible (REVPAR ou "Revenue Per Avallable Room"). Le réseau de distribution au GDS peut permettre aux hôtels d'accroître ces indices de plusleurs façons:

1) aujourd'hul, les agents de voyages font une réservation de chambre pour chaque 10-15 slèges d'avions réservés. Une utilisation accrue du GDS par les agents pourratt permettre d'accroitre le taux d'occupatlon et par conséfuuent la profitabllité d'un hotel;

2) le GDS constutue également un outu eflicace et peu cốteux pour promouvolr une offre auprès des agents de voyages et ainsl générer du revenu adilitlonnel:

3) au fll des années, la facilité d'utilisation de ces outils sest grandement améllorée. diminuant alnsi le temps requis pour faire la réservation et lalssant plus de temps pour la vente des services de l'hôtel;

4) l'apparition de logiclels facilitant la gestion de la cllentèle (base de données-cllents, programmes de fidélisation. marketing direct, etc.) permettent d'accroitre le revenu par cllent, et de mettre sur pled des promollons clblées pour combler les pérlodes plus creuses:
5) enfln, l"utilisation d'outlls technologlques telles les unltés Ilbre-service peimettant un enreglstrement et un départ plus rapide ont un impact certain sur la satisfaction de la cllentèle et ultimement sur le revenu.

Les technologies de l'information ont également une incldence sur les coutts. En utilisant tou|ours l'exemple d'un lottel, en volel quelques Illustrations:

1) Un système de gestion de l'etablissement (“Property Management System" ou PMS) permettra d'automatiser un grand nombre de fonctions et alnsl falre un melleur usage du temps du personnel.

2) De plus, en intégrant ce PMS aux autres systèmes de l'hôtel tels les points-de-vente, les systèmes téléphonlques et le département des ventes et banquets, II permettra une gestlon plus Intégrée et serrée des opérations en lleu et. place d'une gestion de départements autonomes.

3) $70 \%$ à $80 \%$ des réservations de chambres effectuées par les agences de voyages sont faltes directement par téléphone. Une plus grande utilisation d'un GDS par celles-cl permettralent aux hôtellers de rédulre leurs coûts par réservatıon.

\section{Oue nous réserve l'avenir?}

Les technologles de l'Information représentent quelques défls et possiblitiés pour certalns Intervenants de l'industrle. Lavènement de camaux de distribution alternatlfs tels Internet, America Onlne el Prodligy, permettra aux consommateurs de falre leurs sélectlons et réservatlons dilrectement. auprès des foumlsseurs de services. Certalns prodults tels TravelWeb de Thisco sont délà disponibles. Cela pourralt. rédulre à plus ou molns long terme le nombre d'intermédlalres, et forcer une révision de la mission de ces firmes.

De plus, les conts requls pour malntenlr une liste complète d'liôtels Indépendants dans leur base de données pourralent. Inclter les réseaux de distributlon (GDS) à se concentrer sur les hôtels ayant une centrale de réservation. privant ainsl les Indépendants d'un rayonnement plus grand. Pour contrer cette tendance, plusleurs assoclations d'hôtels se sont formées et dotées de leur propre CRS. II en va de méme pour certalnes assoclations tourlstiques qul y ont vu l'opportunltét de fournir une valeur ajoutée additionnelle à leurs membres ou leur région.

Enflin, une tendance à la centrallsation semble se développer dans les clâines hôtellères. En plus de la centrale de réservatlon, d'autres services tels les programmes de fldéllsation, les bases de données-cllents, la gestlon des comptes pour les agences de voyages, etc., serallent centrallsés. Une utillsatlon optimale de la technologle est un pré-requls pour réallser cet objectif.

Lobstacle numéro un demeure la formation du personnel. Un programme de formation permettant aux employés d'apprendre ả leur propre rythme et, si posslble sur place est primordlial. Le programme dolt non seulement expliquer au personnel comment utillser les outils dans le cadre de leurs fonctlons, mals aussl leur permettre d'apprécler en quol ceux-cl contribuent a loblectif ultime de l'industrle: un cllent saltisfait. $f$ 